\title{
On the role of soft computing in modelling the spread of infectious diseases
}

\author{
Nick J. Pizzi \\ Department of Computer Science, University of Manitoba, Winnipeg, Canada
}

\begin{abstract}
Infectious disease modelling is an interdisciplinary activity that has become more prevalent over the last decade as a valuable and practical tool for public health experts. Soft computing, with its suite of techniques and strategies, which effectively handles imprecise, approximate, and vague scenarios, can play an important role in expanding the use of these models. We present a review of soft computing techniques that have been used in infectious disease modelling as well as a prescription of future challenges and issues this discipline must address to be fully accepted by the public health community.
\end{abstract}

Keywords: infectious disease modelling; soft computing; epidemiology; fuzzy sets; dynamical systems; agent-based models; public health

\section{Introduction}

From the Black Death of 1348-1350 [1] and the Spanish flu of 1918-1920 [2], to the recent SARS outbreak of 2003 [3] and the H1N1 influenza pandemic of 2009 [4], infectious diseases have caused significant morbidity, mortality, and socio-economic upheaval. Advanced modelling technologies, which incorporate the most current knowledge of virology, immunology, epidemiology, and vaccines, have recently been developed to identify effective mitigation strategies, and are being increasingly used by public health professionals in the study of both epidemiology and pathogenesis. Tracing its historical roots from the pioneering work of Daniel Bernoulli on smallpox [5] to the classic compartmental approach developed by Kermack and McKendrick [6], infectious disease modelling has evolved to deal with data that are more heterogeneous, less coarse (based at a community, neighbourhood, or individual level), and more complex (joint spatial, temporal and behavioral interactions).

Given the imprecision and vagueness of some of the parameters and epidemiological data used in infectious disease modelling, soft computing techniques and strategies have been exploited to address many of these issues [7][8][9][10]. These run the gamut of fuzzy differential equations to describe nonlinear dynamical systems, fuzzy versions of health risk estimators, multiobjective optimization methods using evolutionary computation to tune system outputs, fuzzy numbers replacing real-valued scalars for disease model input parameters, and an assortment of hybridized combinations.
We have two aims for this paper: present an overview of some soft computing approaches that have been used in infectious disease modelling; and, offer a prescription of the requirements for future investigations to advance, consolidate, and expand the role of soft computing to ensure that these techniques are fully accepted and embraced within the public health community. Section II presents two conventional approaches to infectious disease modelling: compartmental and agentbased. Section III provides a review of some soft computing approaches and extensions to classical modelling. Section IV offers a prescription for future soft computing modelling activities followed by summary remarks in Section V.

\section{Infectious disease modelling}

Over the past decade, infectious disease modelling, simplifying abstractions that use mathematical or computational language and concepts to describe the transmission of an infectious disease, has played a more prevalent role in the decision-making processes of public health experts. Modelling has been used to improve healthcare outcomes through the evaluation of mitigation strategies, the identification of key factors that determine public health policy requirements, informing and optimizing policy decisions, and the more effective application of control measures. These improvements have been achieved through the provision of quantitative predictions, making the underlying assumptions clear and explicit, and increased understanding of disease mechanisms.

\subsection{Compartmental models}

In this section, we present an overview of compartmental models, which model diseases that develop suddenly and then disappear just as suddenly without infecting the entire population, developed in 1927 by Kermack, a public health physician, and McKendrick, a biochemist. Thorough discussions of compartmental models and their extensions may be found in $[11][12][13][14][15]$. This type of model assumes that the populations are homogeneous and fall into one of several "compartments" (classes), for example: "susceptibles" $(S)$, individuals susceptible to the disease but are not infected; "infectives" $(I)$, infected individuals that are able to spread the disease, by contact, with susceptibles; and "recovered" $(R)$, individuals that have recovered (see Fig. 1). As opposed to an epidemiological or human perspective, modelling often considers $R$ to include: isolation from the rest of population; im- 
munization against infection; recovery from the disease with immunity against reinfection; or death caused by the disease.

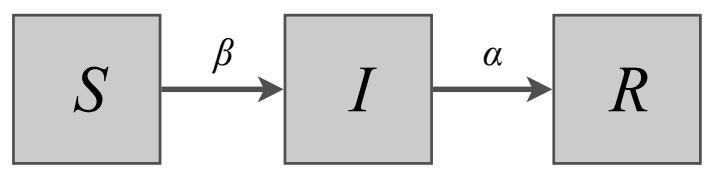

Fig. 1: Schematic of a typical compartmental model.

Compartmental models are formulated in terms of flow of individuals of the population from one compartment to another. An average infective individual of the population makes contact sufficient to transmit infection with $\beta N$ other individuals per unit time, where $N$ represents the total population size $(S+I+R=N)$ and $\beta$ is the contact number. Furthermore, a fraction, $\alpha$, of infectives leave compartment $I$ per unit time $(1 / \alpha$ is the mean infective period). Finally, there is no entry or departure from the population $(d N / d t=0)$ : in other words, the time scale of the disease transmission is much faster than the time scale of births and deaths. This model can be represented as a system of three ordinary differential equations:

$$
\frac{d S}{d t}=-\beta S I, \quad \frac{d I}{d t}=(\beta S-\alpha) I, \quad \frac{d R}{d t}=\alpha I
$$

With the infectious disease model described by (1), we can now define the basic reproductive number, $R_{0}$, the number of secondary infections caused by a single infective introduced into a wholly susceptible population of size $N$

$$
R_{0} \approx \beta N / \alpha
$$

Let us make the reasonable assumption that at the start of an outbreak, $S(0) \approx N$ and $I(0) \approx 0$. Now, if $R_{0}<1$ then the rate of infection is less than the rate of recovery (see (2)), so there is no chance of an epidemic. However, if $R_{0}>1$, then the rate of infection is greater, so an epidemic would ensue.

As this model assumes that there is homogeneous mixing between the compartments, it is not a good description of the start of an infectious disease outbreak. In this case, it may be more realistic to use a network model [16][17] to trace the spread of infections through a population to better examine individual-to-individual contacts. A description of the fundamentals of network models for infectious disease transmission may be found in [18].

Infectious diseases may be modeled using additional compartmental structures (cf. [19]) that may include individuals that have been exposed to the virus but are not yet able to transmit infection, symptomatic versus asymptomatic infectives, and hospitalized individuals. These options and others may be represented using variations of the Kermack-McKendrick model. For exam- ple, a model that incorporates the time since becoming infected may be represented as

$$
\begin{aligned}
& \frac{d S}{d t}=-\beta S(t) \varphi(t) \\
& \varphi(t)=\varphi_{0}(t)+\int_{0}^{t} \beta S(t-\tau) \varphi(t-\tau) A(\tau) d \tau
\end{aligned}
$$

where $\varphi(t)$ is the total infectivity at time $t$ of all infectives within the population, $\varphi_{0}(t)$ represents the total infectivity at time $t$ of all individuals who were previously infected at $t=0$, and $A(\tau)$ is the mean infectivity of all individuals who were infected $\tau$ time units earlier. In this case, $R_{0}$ is

$$
R_{0}=\beta N \int_{0}^{\infty} A(\tau) d \tau
$$

While elegant, this expression's solution is nontrivial (cf. [20]). Finally, the Kermack-McKendrick model may be extended by introducing stochasticity whereby processes act on a set of random variables [21].

\subsection{Agent-based models}

An agent-based model consists of lattice-distributed agents (autonomous decision-making entities) that interact with each other unveiling the underlying dynamics of the system under investigation [22]. These models are used to analyze complex interdependencies with data at finer levels of granularity [23]. For instance, in the case of infectious diseases, agents may represent individuals that have specific levels of susceptibility to the disease in question caused by differences in factors such as lifestyle, prior health conditions, immune system, and so on. The flexibility of agent-based models of infectious diseases permits an effective representation of the complementary interactions between individuals characterized by localized properties and populations at a global level.

For agent-based infectious disease models, an agent comprises state variables that include not only personal characteristics and behavioral traits but also its relationship with the environment and other agents within specified time periods. Demographic data is used to create the agents so that the resulting in silico population follows the same distributions as the physical, real-world population.

The assumptions based on global community parameters have a direct influence on some of the local individual parameters. For instance, with an SIR scenario, a particular agent can be in only one of three states (susceptible, infectious, or recovered). Urban centre characteristics such as commuting distance, demographic density, and city boundaries, may affect the spread of the infectious disease under investigation, as well as agent behaviour. These various mobility patterns can be used to define lattice properties of the infectious disease model. 


\section{Soft computing approaches}

In the case of compartmental modelling approaches such as SIR, differential equations are used to model the spread of an infectious disease within a homogenous population. Investigators have made great inroads in the incorporation of fuzzy set concepts into differential equations through new theoretical approaches and numerical solutions of fuzzy differential equations [7][24][25][26][27][28].

The evolution of a classical (crisp) continuous system depends on a system of ordinary differential equations. It is typical for such a system to have some parameters whose values are only imprecisely known. This imprecision may be represented as a fuzzy number, which produces a system of fuzzy differential equations whose solution can be described by a fuzzy trajectory [10]. By taking a cut through the trajectory for each fixed value, one obtains a fuzzy number, and the uncertainty can be shown as the trajectory of the bases (alpha zero cut) of the solutions. In [29], the authors investigate the SI epidemiological model with a set of interactive fuzzy parameters.

In [30], fuzziness is introduced into spatial susceptible-infected epidemic models [31] through the use of a coupled-map lattice thereby completely bypassing the use of ordinary differential equations. This approach is particularly useful when input parameters such as the initial outbreak are not precisely known. An infectious disease epidemic may be modeled (in a spatiotemporal context without reproducing susceptible and infected individuals) as sweeping through a set of irregular polygons:

$$
\left\{\begin{array}{l}
S_{j}^{t+1}=S_{j}^{t}-S_{j}^{t} \sum_{P_{k} \in N\left(P_{j}\right)} w_{j k} G\left(\mathbf{I}_{j}, d_{j k}\right) I_{k}^{t} \\
S_{j}^{t+1}=I_{j}^{t}+S_{j}^{t} \sum_{P_{k} \in N\left(P_{j}\right)} w_{j k} G\left(\mathbf{I}_{j}, d_{j k}\right) I_{k}^{t}
\end{array}\right.
$$

where $S_{j}^{t}$ and $I_{j}^{t}$ are the respective fuzzy intervals of susceptible and infected individuals within polygon $P_{j}$ at time step $t$ and $G$ describes the connectivity characteristics of the epidemic spread, $d_{j k}$ is the graph distance between the vertices $j$ and $k$ of $P_{j}$ and $P_{k}, N\left(P_{j}\right)$ is the set of polygons in which infected individuals can infect $I_{j}^{t+1}, w_{j k}$ is a weighting factor, and $\boldsymbol{I}_{j}$ contains information about $P_{j}$.

As mentioned in Section II, compartmental models assume that the populations are homogeneous. Heterogeneity can be introduced by allowing the infectious disease to spread strictly along the links of a contact network [18]. In [32], evolutionary computation [33] is used to construct contact networks, through local expansions using a linear chromosome, that identify the maximum duration of the spread of a disease as well as the maximum number of infected individuals. Additionally, the authors specify a useful testing protocol for these networks.

Five main sources of heterogeneity exist in models of infectious disease spread: variability in infectivity; variability in susceptibility; age breakdown; varying contacts; and varying contact distribution across subpopu- lations [34]. As populations are assumed to be homogeneous in standard compartmental models, the contact rate, $\beta$, is a scalar constant. In [35], $\beta$ becomes a fuzzy number

$$
\beta(v)=\left\{\begin{array}{cc}
0 & v<v_{\min } \\
\frac{v-v_{\min }}{v_{M}-v_{\min }} & v_{\min } \leq v \leq v_{M} \\
1 & v_{M}<v<v_{\max }
\end{array}\right.
$$

where $v_{\min }$ is the minimum viral amount for infectious disease transmission, $v_{\max }$ is the maximum viral amount for a given individual and $v_{M}$ is the viral amount that guarantees the transmission of the infectious disease. The authors further generalize their approach by using a linguistic variable, $V$, to deal with smaller groups of individuals with associated fuzzy numbers [36]. Further explorations and refinements to this approach may be found in [37] and [38], which describe an epidemiological model using linguistic variables and fuzzy rules constructed using the extension principle [39].

Relaxing the homogeneity constraint of the compartmental model still further, social network analysis [40] can be used to model specific parameters of disease spread between subpopulations represented without a graph structure. Subpopulations (nodes) that are geodesically near each other have greater mutual influence than distant ones. Fuzzy graphs can be used to expand the functionality of these social networks; however, they suffer from a combinatorial explosion as the number of vertices increases, thereby making it difficult to glean results in a timely fashion. In [41], this issue is addressed by the use of a novel fuzzy binary operation that consolidates information across different fuzzy subgraphs.

In [42], soft computing techniques are applied to the treatment of viral infections using drugs that stimulate the human immune response. The authors extend the standard formulation of the behaviour of immune systems in order to model the pathological consequences of an infection and the side effects of drug treatments. This is accomplished by using a fuzzy set based performance index, which represents imprecise subjective quantities such as patient well-being, that is determined using a sophisticated multi-criteria optimization technique [43].

A nonlinear infection dynamics model is represented in [44] as a set of Mamdani fuzzy controllers [45] that are designed using a genetic-fuzzy system approach. This approach produces fuzzy rules such as "If Viral_Load is Medium and T_Cells_Count is Low then PI_Efficacy is High and RTI_Efficacy is High" where PI (protease inhibitor) is a drug that blocks the chemical mechanisms behind a virus' DNA reproduction and RTI (reverse transcriptase inhibitor) is a drug that reduces the infection rate of CD4+ $\mathrm{T}$ cells (a specific type of immune cell). The utility of this approach lies within its interpretability and control considerations, as it was possible to suggest a specific drug regimen for treating 
an early-detected infection. An analogous approach may be found in [9] that describes a fuzzy rule-based system that extends the classical macroscopic model for HIV and also provides a set of intuitively appealing fuzzy rules that preserves the biological nature and meaning of the transference rate of the HIV virus into an acquired immune deficiency syndrome. The fuzzy expectancy of a symptomatic population is epidemiologically realistic.

With the expanding use of GIS technologies in infectious disease modelling, soft computing approaches are being used to augment conventional analysis techniques; for example, [46] uses a fuzzy expert system with GIS data to make health risk predictions relating to cholera outbreaks. There are also epidemiological studies that have replaced indicator variable in statistical regression models with corresponding fuzzy membership functions [47]. Evolutionary computation is used in [48] to identify the source of infectious disease outbreaks.

\section{Prescription}

Recent public health workshops [4][49], which brought together public health experts, policy makers, and infectious disease modellers, were held to identify the strengths and weakness of disease models and suggest ways to improve their predictive ability to influence public health policy effectiveness. Public health planning requires involvement of communities across disciplines with a firm commitment to the notion that research must ultimately influence policy. Uncertain model inputs that are extremely influential in determining the best course of action should be prioritized for future research investigations. Knowledge translation activities are indispensable to overcome the challenges of community health in an interdisciplinary environment by forging strong links between theory, policy and practice [50].

Given the recent experiences with disease outbreaks, public health experts need rapid and robust decisionmaking as is potentially achievable through modelling approaches. Two challenges to the complete acceptance of these technologies are the timely deployment of models during an infectious disease outbreak and difficulty dealing with scenarios and parameters based on "real-world" data. We have seen that soft computing approaches can help deal with the latter challenge but they must do so not at the expense of the former. The methods we have reviewed in the previous section have solid theoretical underpinnings or good experimental evidence of their utility. However, to move from "theory" to "practice", soft computing based modelling of infectious diseases must satisfy three criteria: reliability, computational efficiency, and adaptability to practical considerations and changes to fundamental knowledge. Further, they must operate with traditional uncertainty and sensitivity analysis methods: Monte Carlo analysis, amplitude sensitivity, random and stratified Latin hypercube sampling [51], regression and correlation analysis, and analysis of stochastic versus subjective uncertainty. An important consideration will be the incorpo- ration of joint spatial and temporal characteristics [29]. Another key area of research will be dealing with imprecise data acquired from disparate database repositories at varying levels of information granularity that may be tackled with the latest soft computing approaches in collaborative clustering and granular computing [52][53].

While compartmental models are useful, with more diverse requirements, finer specificity, and increased expectations by public health experts, agent-based models will play a greater role in infectious disease modelling. However, with flexibility comes complexity; hence, the software implementation of an agent-based model demands more stringent software design requirements than conventional (and simpler) models of the spread and control of infectious diseases, especially with respect to outcome reproducibility, error detection and system management. Outcome reproducibility is a challenge because emergent properties are not analytically tractable, which is further exacerbated by subtle and difficult to detect errors in algorithm logic and software design.

Past software engineering experience [54] and recent literature [55][56][57][58] suggest several guidelines to which agent-based model development should adhere: spiral development methodology; version control; careful code reviews; validation; system profiling; and system determinism. In contrast to compartmental models, which are in general easier to communicate and analyze, agent-based model descriptions are frequently incomplete and therefore less accessible to the reader. In an attempt to tackle this issue, a group of modellers proposed a standard and detailed protocol for describing agent-based models [59]. Achieving these objectives will make agent-based models a much more reliable scientific tool for investigations. "Real world" systems, whether or not soft computing based must take this matter under careful consideration.

\section{Summary}

Realistic infectious disease modelling must incorporate parameters aggregated from disparate database sources; however, these data may be incomplete, imprecise, insufficiently specific, or collated at varying levels of information granularity. Even with extensions, the classical compartmental model fails to adequately capture the nuances of an infectious disease outbreak such as differences in infection probability, age-specific vulnerability, highly heterogeneous spatial properties, and varying contact rates among subpopulations. Further, sensitivity analysis of the model's many parameters must be accomplished in a reliable and timely fashion to ensure that outcome predictions are evaluated and incorporated into public health decision-making. Finally, public health experts tend to think about mitigation strategies for infectious disease outbreaks in a "rule based" manner. With these points in mind, soft computing can readily continue to expand into the practical domain of infectious disease modelling (especially through extensions and enhancements to agent-based 
modelling strategies) and become fully incorporated into the public health discourse.

Concerning sound model representations, investigators of infectious disease spread must keep in mind a broad set of issues: the behaviours and population dynamics that should emerge from the model's representation of important processes versus those being imposed on the model as empirical relations; the adaptive processes to be modeled given the temporal and spatial scales that are to be used; the fitness measures to be used; the necessary user interfaces to make the model outcomes observable and testable; and the development strategies behind the model's design and implementation to ensure that outcome results are reproducible and valid.

The single greatest deficiency with infectious disease modelling is that models are often developed with only the modellers in mind and not the public health experts (this particularly comes across with issues relating to ease of use). It is imperative that soft computing based approaches to infectious disease modelling not suffer from this deficiency.

\section{Acknowledgment}

We thank Dr. A. Demko for his insightful comments relating to software development aspects of infectious disease modelling, Dr. B. Park for discussions on GIS and spatial epidemiology, and Dr. L. Mostaço-Guidolin for his critique on agent-based modelling and parameter imprecision.

This research investigation was supported in part by the Natural Sciences and Engineering Research Council of Canada (NSERC). We also would like to thank the Canadian Institutes of Health Research (CIHR) and the Mathematics of Information Technology and Complex Systems (MITACS) for their invaluable support of recent public health workshops on infectious disease modelling

\section{References}

[1] J. D. Murray, Mathematical Biology: Volume I. An Introduction, Third Edition, Heidelberg: SpringerVerlag, 2007.

[2] J. K. Taubenberger and D. M. Morens, 1918 influenza: The mother of all pandemics, Emerging Infectious Diseases, vol. 12, no. 1, pp. 15-22, 2006.

[3] J. R. Lingappa, L. C. McDonald, P. Simone, and U. D. Parashar, Wresting SARS from uncertainty, Emerging Infectious Diseases, vol. 10, no. 2, pp. 167-170, 2004.

[4] S. M. Moghadas, N. J. Pizzi, J. Wu, and P. Yan, Managing public health crises: the role of models in pandemic preparedness, Influenza and Other Respiratory Viruses, vol. 3, no. 2, pp. 75-79, 2009.

[5] D. Bernoulli, Essai d'une nouvelle analyse de la mortalité causée par la petite verole, Histoire de l'Académie Royale des Sciences, Memoires, Année, pp. 1-45, 1760.
[6] W. O. Kermack and A. G. McKendrick, A contribution to the mathematical theory of epidemics, Proceedings of the Royal Society London A, vol. 115, pp. 700-721, 1927.

[7] Y. Chalco-Cano and H. Román-Flores, On new solutions of fuzzy differential equations, Chaos, Solitons and Fractals, vol. 38, no. 1, pp. 112-119, 2008.

[8] E. Massad, N. R. S. Ortega, L. C. Barros, and C. J. de Struchiner, (Editors), Fuzzy Logic in Action:

Applications in Epidemiology and Beyond, Studies in Fuzziness and Soft Computing, vol. 232, Heidelberg: Springer-Verlag, 2008.

[9] R. M. Jafelice, L. C. de Barros, R. C. Bassanezi, and F. Gomide, Fuzzy modelling in symptomatic HIV virus infected population, Bulletin of Mathematical Biology, vol. 66, no. 6, pp. 1597-1620, 2004.

[10] L. J. Jowers, J. J. Buckley, and K. D. Reilly, Simulating continuous fuzzy systems, Information Sciences, vol. 177, no. 2, pp. 436-448, 2007.

[11] J. Arino, C. Bauch, F. Brauer, S. M. Driedger, A. L. Greer, S. M. Moghadas, N. J. Pizzi, B. Sander, A. Tuite, P. van den Driessche, and J. Watmough, "Pandemic influenza: modelling and public health perspectives," Molecular Biology and Evolution, vol. 8, no. 1, pp. 1-20, 2011.

[12] D. J. Daley and J. Gani, Epidemic Modelling: An Introduction, Cambridge: Cambridge University Press, 1999.

[13] D. J. D. Earn, A light introduction to modelling recurrent epidemics, In: Mathematical Epidemiol$o g y, \mathrm{~F}$. Brauer, P. van den Driessche, and J. Wu, (Editors), Heidelberg: Springer-Verlag, 2008.

[14] S. P. Otto and T. Day, A Biologist's Guide to Mathematical Modelling in Ecology and Evolution, Princeton: Princeton University Press, 2007.

[15] M. J. Keeling and P. Rohani, Modelling Infectious Diseases in Humans and Animals, Princeton: Princeton University Press, 2008.

[16] J. C. Miller, B. Davoudi, R. Meza, A. C. Slim and B. Pourbohloul, Epidemics with general generation interval distribution, Journal of Theoretical Biology, vol. 262, pp. 107-115, 2010.

[17] M. E. J. Newman, The spread of epidemic disease on networks, Physical Review E, vol. 66, 016128, 2002.

[18] L. A. Meyers, Contact network epidemiology: Bond percolation applied to infectious disease prediction and control, Bulletin of the American Mathematical Society, vol. 44, no. 1, pp. 63-86, 2007.

[19] L. C. Mostaço-Guidolin, N. J. Pizzi, and S. M. Moghadas, A classical approach for estimating the transmissibility of the $2009 \mathrm{H} 1 \mathrm{~N} 1$ pandemic, $\mathrm{Ca}$ nadian Applied Mathematics Quarterly, vol. 19, pp. 185-194, 2011.

[20] F. Brauer, C. Castillo-Chavez, and Z. Feng, Discrete epidemic models, Mathematical Biosciences and Engineering, vol. 7, no. 1, pp. 1-15, 2010.

[21] H. Andersson and T. Britton, Stochastic Epidemic Models and Their Statistical Analysis, Lecture 
Notes in Statistics, vol. 151, New York: SpringerVerlag, 2000.

[22] J. A. Sokolowski and C. M. Banks, Modelling and Simulation Fundamentals: Theoretical Underpinnings and Practical Domains, Hoboken: John Wiley and Sons, Inc., 2010.

[23] B. Bonabeau, Agent-based modelling: methods and techniques for simulating human systems, Proceedings of the National Academy of Sciences, vol. 99, Supplement 3, pp. 7280-7287, 2002.

[24] J. J. Buckley and T. Feuring, Fuzzy differential equations, Fuzzy Sets and Systems, vol. 110, pp. 43-54, 2000.

[25] M. Guo and R. Li, Impulsive functional differential inclusions and fuzzy populations models, Fuzzy Sets and Systems, vol. 138, pp. 601-615, 2003.

[26] V. Lakshmikantham and R. N. Mohapatra, Theory of fuzzy differential equations and inclusions, In $R$ P. Agarwal and D. O'Regan, (Editors), Series in Mathematical Analysis and Applications, vol. 6, New York: Taylor \& Francis, 2003.

[27] D. Vorobiev and S. Seikkala, Towards the theory of fuzzy differential equations, Fuzzy Sets and Systems, vol. 125, pp. 231-237, 2002.

[28] M. Oberguggenberger and S. Pittschmann, Differential equations with fuzzy parameters, Mathematical and Computer Modelling of Dynamical Systems, vol. 5, pp. 181-202, 1999.

[29] Cabral VM and Barros LC. The SI epidemiological model with interactive fuzzy parameters, Proceedings of the Annual Meeting of the North American Fuzzy Information Processing Society, Berkeley, USA, August 6-8, pp. 19-22, 2012.

[30] J. M. Baetens and B. De Baets, Incorporating fuzziness in spatial susceptible-infected epidemic models, Proceedings of the Joint International Fuzzy Systems Association World Congress and European Society of Fuzzy Logic and Technology Conference, Lisbon, Portugal, July 20-24, pp. 201-206, 2009.

[31] A. B. Lawson, Statistical Methods in Spatial Epidemiology, Wiley Series in Probability and Statistics, Chichester: John Wiley and Sons, Ltd., 2001.

[32] D. A. Ashlock and F. Jafargholi, Evolving extremal epidemic networks, Proceedings of the IEEE Symposium on Computational Intelligence in Bioinformatics and Computational Biology, Honolulu, USA, April 1-5, pp. 338-345, 2007.

[33] K. A. De Jong, Evolutionary Computation: A Unified Approach, Cambridge: MIT Press, 2006.

[34] K. J. Rothman, S. Greenland, and T. L. Lash, Modern Epidemiology, Third Edition, Philadelphia: Lippincott, Williams and Wilkins Limited, 2008.

[35] L. C. de Barros, M. B. F. Leite, and R. C. Bassanezi, The SI epidemiological models with a fuzzy transmission parameter, Computers and Mathematics with Applications, vol. 45, pp. 1619-1628, 2003.
[36] E. Massad, N. R. S. Ortega, C. J. de Struchiner, and M. N. Burattini, Fuzzy epidemics, Artificial Intelligence in Medicine, vol. 29, pp. 241-259, 2003.

[37] N. Ortega, L. C. Barros, and E. Massad, Fuzzy gradual rules in epidemiology, Kybernetes, vol. 32, pp. 460-477, 2003.

[38] N. R. S. Ortega, P. C. Sallum, and E. Massad, Fuzzy dynamical systems in epidemic modelling, Kybernetes, vol. 29, pp. 201-218, 2000.

[39] L. A. Zadeh, Outline of a new approach to the analysis of complex systems and decision processes, IEEE Transactions on Systems, Man and Cybernetics, vol. SMC-3, pp. 28-44, 1973.

[40] N. Gilbert and K. G. Troitzsch, Simulation for the Social Scientist, Second Edition, New York: Open University Press, 2005.

[41] P. S. Nair and S. T. Sarasamma, Data mining through fuzzy social network analysis, Proceedings of the Annual Meeting of the North American Fuzzy Information Processing Society, San Diego, USA, June 24-27, pp. 251-255, 2007.

[42] J. A. M. F. de Souza, R. K. H. Galvão, and T. Yoneyama, Optimization of the therapy of viral infections using fuzzy techniques, Proceedings of the American Control Conference, San Diego, USA, June 2-4, pp. 4051-4055, 1999.

[43] G. Chiandussi, M. Codegone, S. Ferrero, and F. E. Varesio, Comparison of multi-objective optimization methodologies for engineering applications, Computers \& Mathematics with Applications, vol. 63, no. 5, pp. 912-942, 2003.

[44] M. A. Melgarejo, C. A. Peña-Reyes, and E. Sanchez, A genetic-fuzzy system approach to control a model of the HIV infection dynamics, Proceedings of the IEEE International Conference on Fuzzy Systems, Vancouver, Canada, July 16-21, pp. 10574-10581, 2006.

[45] E. H. Mamdani, Application of fuzzy algorithms for the control of a simple dynamic plant, Proceedings of the IEE, vol. 121, no. 12, pp. 1585-1588, 1974.

[46] G. Fleming, M. van der Merwe, and G. McFerren, Fuzzy expert systems and GIS for cholera health risk prediction in southern Africa, Environmental Modelling \& Software, vol. 22, no. 4, pp. 442-448, 2007.

[47] A. Bolotin, Replacing indicator variables by fuzzy membership functions in statistical regression models: examples of epidemiological studies, In: Biological and Medical Data Analysis, Lecture Notes in Computer Science, vol. 3337, J. M. Barreiro, F. Martín-Sánchez, V. Maojo, and F. Sanz, (Editors), Heidelberg: Springer-Verlag, pp. 251258, 2004.

[48] T. Jefferson, E. Grossi, and M. Buscema, Powerful computerized spatial epidemiology and semantics through the use of novel mathematical objects: Can artificial intelligence systems identify outbreak sources? Proceedings of the Annual Meeting of the North American Fuzzy Information Processing So- 
ciety, New York, USA, May 19-22, pp. 60205, 2008.

[49] S. Moghadas, T. Day, C. Bauch, S. M. Driedger, F. Brauer, A. Greer, P. Yan, J. Wu, N. J. Pizzi, and D. Fisman, Modelling of pandemic influenza: a guide for the perplexed, Canadian Medical Association Journal, vol. 181, no. 3-4, pp. 171-173, 2009.

[50] S. M. Moghadas, N. J. Pizzi, J. Wu, P. Yan, D. N. Fisman, and S. C. Tamblyn, Canada in the face of the $2009 \mathrm{H} 1 \mathrm{~N} 1$ pandemic, Influenza and Other Respiratory Viruses, vol. 5, no. 2, pp. 83-88, 2011.

[51] J. C. Helton and F. J. Davis, Latin hypercube sampling and the propagation of uncertainty in analyses of complex systems, Reliability Engineering and System Safety, vol. 81, no. 1, pp. 23-69, 2003.

[52] W. Pedrycz, The principles of justifiable granularity and an optimization of information granularity allocation as fundamentals of granular computing, Journal of Information Processing Systems, vol. 7, pp. 397-412, 2011.

[53] D. Graves, J. Noppen, and W. Pedrycz, Clustering with proximity knowledge and relational knowledge, Pattern Recognition Letters, 2013 (in press).

[54] N. J. Pizzi, Software quality prediction using fuzzy integration: a case study, Soft Computing Journal, vol. 12, no. 1, pp. 67-76, 2008.

[55] L. C. Mostaço-Guidolin, N. J. Pizzi, A. B. Demko, and S. M. Moghadas, A software development framework for agent-based infectious disease modelling, In: Biomedical Engineering Trends in Electronics, Communications and Software, A. N. Laskovski, (Editor), Rijeka: InTech, pp. 641-664, 2011.

[56] S. F. Railsback, Concepts from complex adaptive systems as a framework for individual-based modelling, Ecological Modelling, vol. 139, pp. 47-62, 2001.

[57] P. Ormerod and B. Rosewell, Validation and Verification of Agent-Based Models in the Social Sciences, In: Epistemological Aspects of Computer Simulation in the Social Sciences, F. Squazzoni, (Editor), Berlin: Springer-Verlag, pp. 130-140, 2009.

[58] S. F. Railsback, S. L. Lytinen, and S. K. Jackson, Agent-based simulation platforms: review and development recommendations, Simulation, vol. 82, no. 9, pp. 609-623, 2006.

[59] V. Grimm, U. Berger, F. Bastiansen, S. Eliassen, V. Ginot, J. Giske, J. Goss-Custard, T. Grand, S. K. Heinz, G. Huse, A. Huth, J. U. Jepsen, C. Jørgensen, W. M. Mooij, B. Müller, G. Pe'er, C. Piou, S. F. Railsback, A. M. Robbins, M. M. Robbins, E. Rossmanith, N. Rüger, E. Strand, S. Souissi, R. A. Stillman, R. Vabø, U. Visser, and D. L. DeAngelis, A standard protocol for describing individual-based and agent-based models, Ecological Modelling, vol. 198, no. 1-2, pp. 115-126, 2006. 